\title{
Tratamento das hérnias inguinais: Bassani ainda atual? Estudo randomizado, prospectivo e comparativo entre três técnicas operatórias: Bassini, Shouldice, McVay
}

\author{
W.E.M. Mittelstaedt, A.J . Rodrigues J R., J .Duprat, R.G. Bevilaqua, D. Birolini \\ Disciplina deCirurgia Geral edoTrauma do Hospital das Clínicas da Faculdade de Medicina da Universidade de SãoPaulo, SãoPaulo, SP.
}

\begin{abstract}
RESUMO - OвJ etivo. Comparar os resultados tardios (recidivas) obtidos com o emprego de três técnicas operatórias para correção das Hérnias Inguinais no adulto: Bassini, Shouldice e Mc Vay.

Casuística e Método. Foram analisados os resultados obtidos em 119 pacientes adultos portadores de hérnias inguinais, alguns com hérnias bilaterais (totalizando 136 hérnias), operados por uma das três técnicas seguintes: Bassini, Shouldice e McVay. A maioria dos pacientes foi do sexo masculino $(93,3 \%)$. O estudo foi prospectivo, randomizado, com distribuição uniforme dos três ti pos de hérnias (indi reta, di reta e mista) entre os três grupos de técnicas operatórias. Foi avaliado o número de recidivas observadas durante um período de quatro anos. Estes resultados foram submetidos à análise estatística através de curvas de sobrevida de Kaplan-Mayer .

Resultados. Oito hérnias operadas pela técnica de Bassini recidivaram neste período, três no grupo Shouldice e duas operadas a McVay. Dentre os operados a Bassini, os piores resultados foram
\end{abstract}

\section{NTRODUÇÃO}

As estruturas anatômicas na região inguinal se dispoem de tal forma, que deixam uma área pouco protegida contra a pressão intra-abdominal. Esta área que Fruchaud denominou de "óstio miopectíneo" ${ }^{\prime \prime}$, é recoberta e protegida tão somente por uma fina camada de tecido fibroso denominado de "Fáscia Transversal " (FT). Acrescente-se a isto o fato das estruturas fasciais, aponeuróticas e musculares sofrerem com o avançar da idade, um processo natural de envel hecimento e enfraquecimento progressivos. A quantidade e qualidade do colágeno e das fibras elásticas dos tecidos nesta região se alteram, tornando tais pessoas mais propensas ainda ao aparecimento de hérnias ${ }^{2-6}$. Estudos recentes apontam, além do mais, também para uma provável influência genética nestas alterações ${ }^{7}$. A hérnia inguinal é conseqüência justamente da falência deste e de outros mecanismos de contenção observados nas hérnias diretas $(29 \%$ de recorrência) quando comparados aos obtidos com as hérnias indiretas (16\%). Os resultados gerais projetados para quatro anos de seguimento, mostram diferenças estatisticamente significantes entre Bassini e Shouldice: $35,7 \%$ vs $23,7 \%$ mesmo foi observado na comparação entre Bassini e McVay: $35,7 \%$ vs $\mathbf{8 , 5 \%}$. As diferenças entre os grupos Shouldice e McVay não foram significantes.

Conclusão. $O$ índice de recidiva de $35,7 \%$ para as hérnias operadas pela técnica de Bassini foi surpreendente, obrigando-nos a suspender o estudo. Há um índice excessivamente alto de insucesso após a utilização da técnica de Bassini, que foi durante um século o método mais usado para correção de hérnias inguinais no mundo inteiro. Os métodos de Shouldice e de McVay, embora de execução técnica mais complexa, devem ser as preferidos quando se opta pela utilização de técnicas convencionais de herniorrafia.

UNITERMOS: Hérnia. Hérnia inguinal.

de vísceras, com a conseqüente extrusão das mesmas ou de gordura preperitoneal por esta região.

$\mathrm{O}$ tratamento das hérnias inguinais é eminentemente cirúrgico. Mais de uma centena de técnicas operatórias tem sido propostas para sua correção, desde que Marcy, em $1871^{8}$, e Bassini, em 1884, lançaram as bases, até recentemente consideradas racionais, deste tratamento. Apesar desta enorme variedade de opções, os insucessos de tratamento com recidiva das hérnias continuam freqüentes demais. N os EUA operam-seem média 500.000 hérnias anual mente, das quais 100.000 são recidivas ${ }^{10}$. Este é o motivo pel o qual nenhuma técnica isolada tenha conseguido convencer definitivamente os cirurgiões a adotá-la universalmente $\mathrm{e}^{11}$.

Existem preferências pessoais por determinadas técnicas operatórias, baseadas geralmente na experiência de cada cirurgião. Uma análise objetiva e impessoal dos resultados a longo prazo, entretanto, é prejudicada principalmente pelas dificuldades de 
seguimento destes pacientes. Análises retrospectivas são fal has e poucos são os estudos prospectivos e randomizados comparando as diferentes técnicas.

O princípio clássico introduzido por Bassini no final do século passado, consiste em se corrigir a hérnia inguinal através da reaproximação e sutura de estruturas músculo-aponeuróticas deiscentes e enfraquecidas, reconstituindo de maneira anátomo-funcional a região inguinal. O método de Bassini foi, durante o transcorrer do século $X X$, o mais utilizado universal mente para o tratamento desta doença. Das inúmeras variantes propostas a esta técnica inicial, duas se destacaram na aceitação dos cirurgiões no mundo inteiro: a técnica de McVay, difundida a partir de 1945 e a de Shouldice a partir de 1960.

A introdução de próteses de polipropileno no tratamento das hérnias inguinais, iniciada em 1959 por Usher nos EUA ${ }^{12-14}$ e em 1969 por $\mathrm{Falci}^{15,16}$ em nosso meio, ganhou maior popularidade nos últimos dez anos após as publicações de Lichtenstein e sua escola ${ }^{10}$.

Criou-se um conceito novo, denominado de técnicas sem tensão (tension free), pel o qual a reconstrução do trígono inguinal se faz pela sobreposição de material protético em toda sua área, sem suturas de aproximação. As técnicas video-laparoscópicas mais recentes se apoiam neste mesmo princípio.

As técnicas clássicas, entretanto não perderam seu lugar, principalmente naqueles casos em que a utilização de próteses não é desejada, como nos pacientes jovens e mulheres por exemplo.

Preocupados em escol her um método operatório padrão a ser a ser adotado por nossos residentes, decidimos, há cerca de cinco anos, iniciar um estudo prospectivo, randomizado, comparando três técnicas clássicas de herniorrafia inguinal: Bassini, Shouldice e McVay.

A apresentação destes resultados e a discussão dos mesmos é o objeto deste trabalho.

\section{CASUÍSTICA E MÉTODO}

Entre março de 1992 e março de 1996, foram al ocados 119 pacientes portadores de hérnia ingui nal que procuraram o ambulatório de Cirurgia Geral do Hospital das Clínicas da FMUSP. Eram pacientes adultos, de ambos os sexos e o único critério de seleção foi o de terem residência fixa na área de São Paulo pelo menos há cinco anos, afim de garantir um seguimento pós-operatório razoável. Foram 119 pacientes, dos quais 17 com hérnias bilaterais, totalizando 136 hérnias operadas. A grande maioria era do sexo masculino (111 pacientes),

\begin{tabular}{|lccc|}
\hline \multicolumn{3}{|c|}{ Tabela 1 - Tratamento das hérnias inguinais no adulto } \\
\hline \multicolumn{3}{|c}{ Estudo comparativo } \\
\cline { 2 - 4 } $\begin{array}{l}\text { Técnicas } \\
\text { Analisadas }\end{array}$ & Total & Unilaterais & Bilaterais \\
McVAY & 50 hérnias & $34-68 \%$ & $16-32 \%$ \\
Bassini & 44 hérnias & $31-70,5 \%$ & $13-29,5 \%$ \\
Shouldice & 42 hérnias & $37-88,1 \%$ & $5-11,9 \%$ \\
\hline Obs.: São 119 pacientes - 17 com hérnias bilaterais \\
\hline
\end{tabular}

distribuídos de maneira uniforme por todas as faixas etárias entre 18 e 70 anos. Sessenta pacientes $(50,4 \%)$ eram portadores de hérnias à direita, $42(35,3 \%)$ do lado esquerdo e $17(14,3 \%)$ bilaterais. Oitenta e cinco $(62,5 \%)$ hérnias eram do tipo indireto ou do tipo II segundo classificação de Nyhus (17), 41 (30,24\%) se apresentaram como hérnias diretas ou do ti po III A e 10 (7,3\%) foram do tipo misto ou IIIB.

A pós a internação, os pacientes eram submetidos à randomi zação al eatória que definiu a técnica operatória a ser adotada. Desta forma 50 hérnias foram corrigidas segundo técnica de McVay, 44 conforme Bassini e 42 a Shouldice. No primeiro grupo (McVay) 16 hérnias (32\%) foram bilaterais e $34(68 \%)$ unilaterais. No grupo Bassini 13 hérnias $(29,5 \%)$ foram bilaterais e 31 (70,5\%) unilaterais. No grupo Shouldice 5 (11,9\%) foram bilaterais e 37 $(88,1 \%)$ unilaterais (Tabela 1$)$.

A distribuição dos diferentes tipos de hérnias nos três grupos de técnica operatória foi uniforme, não havendo diferenças estatisticamente significantes entre estes números. A escol ha do tipo de anestesia foi sempre deixada a cargo do médico anestesista. Assim, 67 pacientes foram operados em raquianestesia, 38 em bloqueio peridural e 14 em anestesia geral. Não foi empregada em nenhum caso antibióticoterapia profilática.

\section{Técnicas operatórias}

As seguintes etapas for am comuns as três técnicas: incisão oblíqua sobre a região inguinal; secção e divulsão da tela subcutânea através de suas 3 camadas: túnica areolar, fáscia superficial e túnica lamelar, ligando-se os vasos epi gástri cos superficiais e circunflexos ilíacos superficiais; individualização do anel inguinal externo e a partir deste, abertura da aponeurose do m. oblíquo externo. I sol amento do funícul o esper máti co ou ligamento redondo; abertura Iongitudinal da túnica cremastérica e fascia espermática ao longo do funículo; expl oração do seu conteúdo, isol amento e di ssecção do saco herniário quando presente (h.indireta); ligadura e secção do ligamento redondo nas mulheres procurando-se poupar os nervos ilio-inguinal e 
ilio-hipogástrico; ressecção do saco herniário após ligadura alta do mesmo; secção bilateral dos feixes do músculo cremaster junto ao anel inguinal profundo; avaliação da parede posterior do conduto inguinal e do grau de firmeza da fáscia transversal $(\mathrm{FT})$; identificação de eventual hérnia direta; abertura longitudinal da FT em toda sua extensão e exploração do espaço préperitoneal, identificando-se hérnia crural quando presente; reconstituição e reforço da região inguinal por uma das três técnicas escolhidas; fechamento da ap. do m. oblíquo ext. através de sutura continua com fio de poliglactina 000 por cima do funículo; fechamento da tela sub-cutânea e pele; curativo oclusivo.

Técnica de Bassini - aproximação com sutura em tripla camada do músculo oblíquo interno (MOI), do arco aponeurótico do músculo transverso (AAMT) e da FT ao trato ilio-púbico (lig. de Thomson) e ligamento inguinal com pontos separados de nylon 00 por detrás do funículo.

Técnica de McVay - aproximação e sutura do AAMT aoligamento de Cooper com pontos separados de nyl on 0 até a altura dos vasos femorais. Dali até $o$ anel ing. profundo, os pontos são passados unindo a aponeurose ou o músculo transverso ao ligamento de Thomson eligamento inguinal quando a firmeza do primeiro (Thomson) não nos parecia suficiente.

Técnica de Shouldice - imbricação em jaquetão dos dois folhetos criados com a abertura da FT através de dois planos superpostos de sutura contínua com nyl on 000 na seguinte ordem: 1이 planofol heto lateral à base do fol heto medial, este último correspondendo na realidade ao arco aponeurótico do transverso; 20 plano: folheto medial ao ligamento inguinal; 3으 e $\%$ planos: músculos oblíquo interno e transverso ao ligamento inguinal, sendo que o 40 plano é práticamente redundante sobre o 3‥

\section{ANÁLISE ESTATÍSTICA}

Os resultados quanto à recidiva foram analisados por curvas de sobrevida de Kaplan-Mayer. As diferenças entre tais curvas foram avaliadas pelos métodos de Log-Rank e Breslow. Com isto, mesmo sem o seguimento prolongado de todos os pacientes, conseguimos projetar as taxas de recidiva.

\section{RESULTADOS}

Complicações pós-operatórias imediatas: não houve nenhuma complicação sistêmica. Dentre as complicações locais destacam-se as infecções da ferida operatória, que ocorreram em oito casos

\begin{tabular}{|c|c|}
\hline \multicolumn{2}{|c|}{$\begin{array}{l}\text { Estudo comparativo } \\
\text { Seguimento }\end{array}$} \\
\hline Até 6 meses & 32 hérnias \\
\hline de 07 a 12 meses & 21 hérnias \\
\hline de 13 a 24 meses & 27 hérnias \\
\hline de 25 a 36 meses & 16 hérnias \\
\hline de 37 a 48 meses & 26 hérnias \\
\hline de 49 a 51 meses & 14 hérnias \\
\hline Total & 136 hérnias \\
\hline
\end{tabular}

Tabela 3 - Tratamento das hérnias inguinais no adulto

\begin{tabular}{|c|c|c|c|}
\hline \multicolumn{4}{|c|}{$\begin{array}{c}\text { Estudo comparativo } \\
\text { Hérnias recidivadas distribuidas pelas diferentes técnicas }\end{array}$} \\
\hline & Bassini & Shouldice & McVay \\
\hline Indireta & 4 recidivas(16\%) & 2 recidivas $(7 \%)$ & 2 recidivas $(6 \%)$ \\
\hline Direta & 4 recidivas(29\%) & 1 recidiva(9\%) & 0 recidiva \\
\hline Mista & 0 recidiva & 0 recidiva & 0 recidiva \\
\hline Total & 8 recidivas & 3 recidivas & 2 recidivas \\
\hline
\end{tabular}

(5,9\%). Em cinco delas houve necessidade de drenagem ampla da tela sub-cutânea. Destas, três evoluíram para recidiva posterior das hérnias (2 Bassini e $1 \mathrm{McV}$ ay). Em três casos com infecção leve, esta regrediu com antibioticoterapia e cuidados locais. Oito hérnias evoluíram com sero-hematomas pouco volumosos, que regrediram espontaneamente, assim como também as três com edema local. Em um caso no qual foi deixada a porção distal do saco herniário, houve desenvolvimento posterior de hidrocele, que também regrediu sem necessitar de maiores cuidados. Não houve diferença estatisticamente significante entre os três grupos de técnica no tocante a estas compli cações.

Seguimento: (Tabela 2) - Pacientes operados de 14 hérnias puderam ser seguidos por mais de quatro anos (até 51 meses), 26 por 3 a 4 anos, 16 por 2 a 3 anos, 27 por 1 a 2 anos, 21 por 6 meses a 1 ano e 32 só por 6 meses. Todos os pacientes for am seguidos e examinados a cada seis meses por um dos autores (WEMM).

Resultados tardios. Recidivas. (Tabela 3). Houve um total de 13 recidivas; oito no grupo dos operados a Bassini, três no grupo Shouldice e dois no de McVay. A tabela mostra a distribuição destas recidivas nos diferentes tipos de hérnias e nos grupos correspondentes de técnica operatória. Assim, das 25 hérnias indiretas operadas pela técnica de Bassini quatro (16\%) recidivaram, no grupo Shouldice com 29 hérnias - duas (7\%) recidivaram e no grupo McVay com 31 hérnias - duas (6\%). Dentre as 14 hérnias diretas operadas pelo método 


\begin{tabular}{|c|c|c|c|c|c|c|}
\hline \multicolumn{7}{|c|}{ Estudo comparativo } \\
\hline \multicolumn{7}{|c|}{ Porcentagens de pacientes sem recidiva calculadas por curvas de Kaplan - Mayer } \\
\hline \multirow{2}{*}{$\begin{array}{c}\text { Técnicas } \\
\text { Analisadas }\end{array}$} & \multicolumn{2}{|c|}{ Bassini } & \multicolumn{2}{|c|}{ McVay } & \multicolumn{2}{|c|}{ Shouldice } \\
\hline & Unilaterais & Bilaterais & Unilaterais & Bilaterais & Unilaterais & Bilaterais \\
\hline $\begin{array}{l}\text { Porcentual sem } \\
\text { recidiva }\end{array}$ & $60 \%$ & $58 \%$ & $90 \%$ & $94 \%$ & $75 \%$ & $100 \%$ \\
\hline $\begin{array}{l}\text { Tempo máximo } \\
\text { de seguimento } \\
\text { em meses }\end{array}$ & 48 & 44 & 50 & 50 & 51 & 25 \\
\hline
\end{tabular}

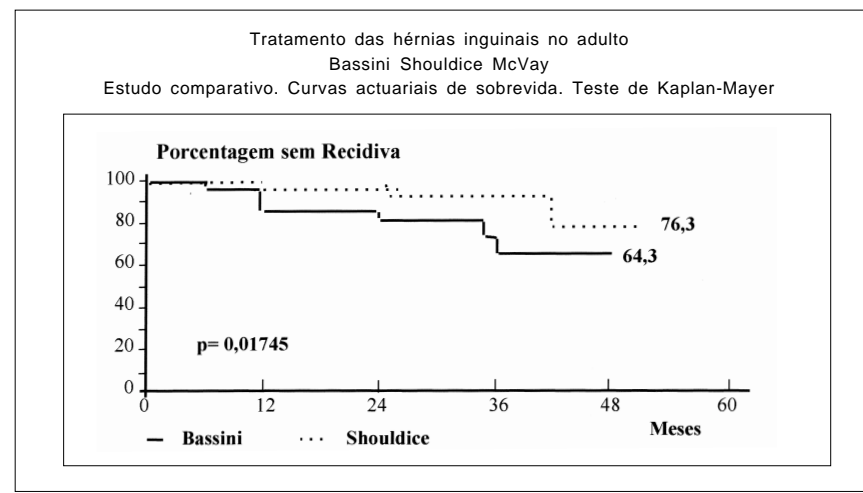

Fig. 1 - Após 4 anos. Taxas de recidiva projetadas: Bassini, 35,7\%; Shouldice, 23,7\%

de Bassini, quatro (29\% recidivaram, de 11 no grupo Shouldice, uma ( $9 \%$ ) recidivou e nenhuma no grupo McVay (16 hérnias). Dentre as hérnias mistas (19 hérnias) distribuídas pelos três grupos de técnica, nenhuma recidivou. Destas recidivas, oito ocorreram de 6 a 12 meses após a herniorrafia primária. Outras cinco recidivas ocorreram após três anos. Não houve diferença estatisticamente significante no que tange ao tempo de seguimento e idade dos pacientes nas três técnicas.

A plicando-se o teste de Kaplan-Mayer para confecção de curvas actuariais de sobrevi da na análise dos porcentuais de recidiva nas três técnicas operatórias, obtivemos os seguintes resultados: na comparação entre Bassini e Shouldice (Figura 1) observa-se que após quatro anos, $76,3 \%$ das hérnias operadas pela técnica de Shouldice e $64,3 \%$ daquelas operadas a Bassini, permaneciam sem recidiva ou seja, 35,7\% daquelas operadas a Bassini recidivaram até quatro anos de seguimento, contra $23,7 \%$ das operadas a Shouldice. Esta diferença é estatisticamente significante. $\mathrm{Na}$ comparação entre Bassini e McVay (Figura 2) esta diferença é ainda mais nítida, ou seja, $91,5 \%$ das hérnias operadas pela técnica de McVay permaneciam sem recidiva contra os $64,3 \%$ do grupo Bassini. Isto equivale dizer que só $8,5 \%$ das hérnias

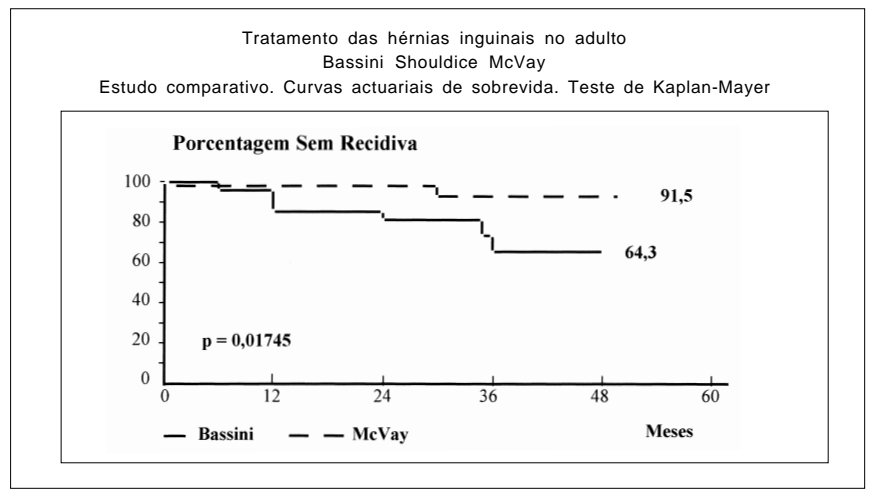

Fig. 2 - Após 4 anos. Taxas de recidiva projetadas: Bassini, 35,7\%; McVay, 8,5\%

operadas a McVay recidivaram no período estimado de quatro anos. As diferenças entre os resultados obtidos pelas técnicas de Shouldice e McVay, quando confrontadas (Figura 3), não são estatisti camente significantes $(p=0,374)$. A figura 4 mostra os resultados comparativos obtidos com as três técnicas (estatisticamente significantes).

A tabel a 4 mostra os resultados tardios observados considerando-se isol adamente as hérnias unilaterais e bilaterais. A anál ise dos porcentuais sem recidiva ao fim dos tempos máximo de seguimento nas três técnicas não evidencia diferenças estatísticamente significantes entre hérnias unilaterais e bilaterais.

\section{DISCUSSÃO}

A maior incidência de hérnias ingui nais no sexo masculino (93,3\% em nosso estudo) é um aspecto inter essante e já apontado por outros autores ${ }^{18}$. A fáscia transversal (FT) é muito mais firme e resistente na mulher. Isto explica a absoluta infreqüência de hérnias diretas na mesma ${ }^{18}$.

Só oito $(6,7 \%)$ pacientes de nosso col etivo eram do sexo feminino e todas portadoras de hérnias indiretas. A maior freqüência de hérnias ínguinocrurais do lado direito $(50,4 \%)$ é provavelmente 


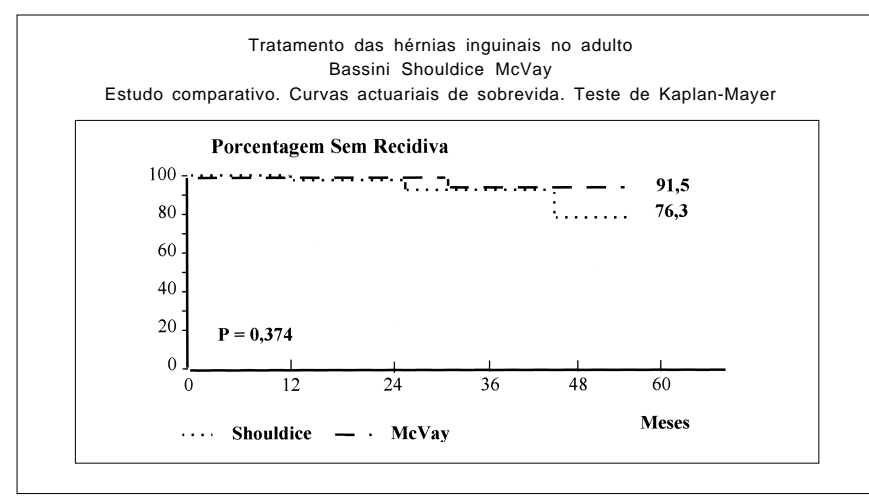

Fig. 3 - Após 4 anos. Taxas de recidiva projetadas: Shouldice, 23,7\%; McVay, 8,5\%

conseqüência da inserção oblíqua da raiz do mesentério, da esquerda para a direita, determinando mai or ação do peso das vísceras neste lado ${ }^{19}$. As hérnias indiretas foram mais freqüentes $(62,5 \%)$ do que as diretas (30,2\%), fato observado na maioria das grandes estatísticas ${ }^{20}$.

Houve uma distribuição uniforme do número total de hérnias operadas e de seus tipos (indireta, direta ou mista) nos três grupos de técnicas .

E doardo Bassini Iançou, no final do século passado, mais especificamente entre 1884 e 1890 na Italia, as bases até recentemente consideradas de forma unânime como racionais do tratamento das hérnias inguinai $\mathrm{s}^{21-24}$. Descreveu a aproximação e sutura em tripla camada do múscul o oblíquo interno (MOI), transverso ( $\mathrm{MT}$ ) e $\mathrm{FT}$ ao ligamento inguinal. Antes de Bassini, com exceção de Marcy, cirurgião americano que em 1870 propôs o estrei tamento isolado do anel inguinal profundo, o tratamento geralmente utilizado limitava-se ao uso de fundas ou somente ao estreitamento do anel inguinal superficial. A técnica de Bassini veio a tornar-se o tratamento padrão e mais utilizado no mundo inteiro até recentemente. Da leitura de seus trabal hos não fica claro um detal he técnico que só 42 anos mais tarde veio à tona quando da publicação de um livro e atlas cirúrgico por seu discípulo Attilio Catterina ${ }^{25}$, que é a abertura ampla da FT em toda sua extensão antes de efetuar-se a rafia do assoalho inguinal.

Das inúmeras modificações introduzidas à técnica original de Bassini, a que ganhou maior destaque na prática foi a utilização do arco aponeurótico do músculo transverso medial mente e do ligamento de Thomson lateralmente. Em nossa experiência, entretanto, a aponeurose do músculo transverso se apresenta muitas vezes como uma estrutura muito frágil e fina ou os MOI e MT se mantêm carnosos até sua inserção na bainha dos múscul os retos ${ }^{10,26}$. I sto tem sido relatado na literatura e provavelmente não foi por outro motivo que

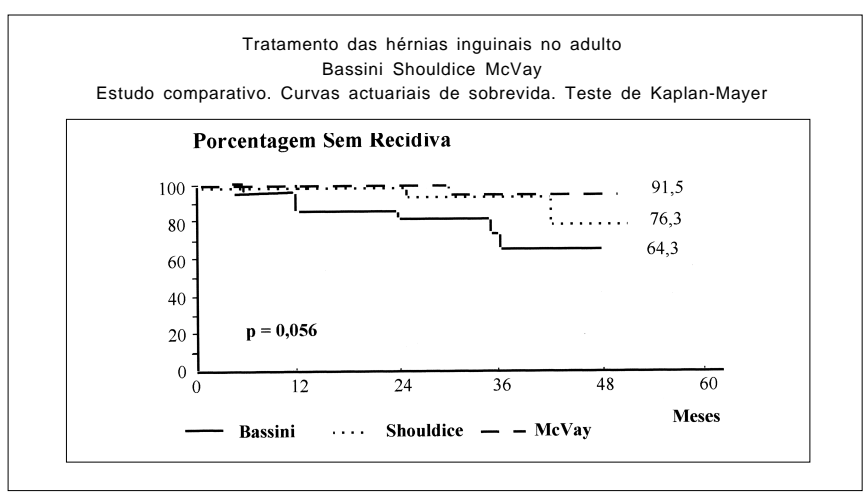

Fig. 4 - Após 4 anos. Taxas de recidiva projetadas: Bassini, 35,7\%; Shouldice, 23,7\%; McVay, 8,5\%.

Rutledge teve que usar adicional mente material de prótese (márlex) em 13,4\% de seus pacientes ${ }^{27}$. Também o trato ílio-púbico (ligamento de Thomson) se apresenta em $75 \%$ dos casos diminuído em sua espessura e resistência ${ }^{28}$. Não praticamos incisões de relaxamento nas herniorrafias a Bassini pois a tensão resultante das suturas não nos pareceu grande.

A utilização do ligamento pectíneo, mais conhecido como ligamento de Cooper, foi referida pela primeira vez em herniorrafias inguinais pelo cirurgião austríaco Georg Lothei ssen em 1897. Seu procedimento em uma série de 12 casos foi publicada em 189829. Esta inovação permaneceu ignorada até que outro cirurgião e anatomista como Bassini, o americano, Chester McVay, propusesse de novo em 1942 a utilização do ligamento pectíneo ${ }^{30}$. Seu argumento foi o de que esta estrutura anatômica era mais firme e fisi ol ógi ca do que o ligamento inguinal uma vez que representava a área de inserção natural da FT. O epônimo de "técnica de McVay" foi universalmente adotado, principalmente nos países de língua inglesa ${ }^{31}$. Enquanto Mc Vay só recomendava seu método operatório para as hérnias diretas, indiretas volumosas, mistas ou crurais $^{32}$, muitos dos seus seguidores e propagadores entusiastas não relutam em indicá-lo para todas as hérnias inguino-crurais no homem assim como na mulher 33 .

Além da fragilidade ocasional da aponeurose do músculo transverso, já assinalada, chamou-nos a atenção que o método de $\mathrm{McV}$ ay é de execução técnica mais difícil e trabalhosa, principalmente em obesos. O ligamento de Cooper situa-se mais distante e profundo, o que acaba determinando suturas mais tensas. A incisão de relaxamento na bainha dos músculos retos passa a ser obrigatória e foi realizada de rotina em nossas casos. Barbier ${ }^{34}$ rel ata que no primeiro ano de herniorrafia por esta técnica, de um total de 1.040 pacientes operados entre 1970 e 1978, chegou a ter 47,37\% de recidi- 
vas. Atribui este elevado índice de insucesso à falta de experiência dos cirurgiões com o método e a não utilização de incisões de relaxamento. A limitação cranial das suturas na técnica de McVay é representada pelos vasos femorais. Dali até o anel inguinal profundo fica uma longa distância que precisa ser tratada as custas de "pontos de transição". Nyhus chama a atenção para o fato de que a assim chamada bainha femoral, âncora lateral das suturas nesta região, é na realidade oligamento de Thomson e não a adventícia destes vasos, cuja pega na sutura seria perigosíssima ${ }^{35}$. Como este ligamento é muitas vezes frágil, acaba-se por usar o ligamento inguinal. Este é, aliás o único método operatório dentre os três que permite corrigir também eventuais hérnias crurais concomitantes.

Outra técnica que sobretudo nas décadas de 1970 e 1980 gozou de amplas simpatias, é a "técni ca canadense" ou "método de Shouldice". F oi idealizada e desenvolvida até sua apresentação atual por E.E. Shouldice e seus colaboradores N. Obney e E. Ryan nos anos de 1950 a $1953^{36}$. O centro mundial de difusão deste método operatório é a Clínica que em Toronto (Canadá) tem o nome de seu fundador (Shouldice), especializada no tratamento de Hérnias.Depois que os primeiros cirurgiões americanos visitaram a Clínica e difundiram a técnica nos EUA no fim da década de $1960^{37,38}$, o método foi sendo paulatinamente adotado no mundo intei ro. Passou a ser reconhecido pela maioria dos "cirurgiões convencionais" como o método padrãoouro para o tratamento das hérnias inguinais ${ }^{39-41}$. Glassow $^{42}$ e Bendavid ${ }^{43}$ têm sido através de suas publicações os maiores difusores desta técnica, embora este último venha manifestando sua simpatia crescente por técnicas "sem tensão" com prótese ${ }^{44}$. Í ndi ces de reci diva surpreendentemente baixos, da ordem de 0,7 a 1,6\%, têm si do rel atados por cirurgiões desta clínica $43,45,46$.

A herniorrafia a Shouldice também é de execução técnica mais difícil do que o Bassini, devido ao fato da FT se apresentar geralmente muito del gada e frágil. Seu imbricamento em dois planos de sutura contínua é trabalhoso e por vezes problemático. Os 3e 4ำplanos de sutura unindo de forma redundante os MOI e MT ao ligamento inguinal, são idênticos à técnica de Bassini , razão pela qual al guns consideram Shouldice um "Bassini modificado". Estes dois últimos planos são considerados por muitos cirurgiões como supérfluos, além de causarem maior desconforto pós-operatório devido as suturas engl obando tecido muscular ${ }^{47-50}$. E m nossos casos realizamos todos os quatro planos da técnica original. Os pacientes deste estudo foram operados em anestesia geral, peridural ou raquidiana.
F oge ao âmbito deste estudo entrar na discussão do mérito da anestesia local na cirurgia das hérnias, mesmo porque não existem estudos randomizados comparando os resultados de herni orrafias com diferentes tipos de anestesi ${ }^{41}$. Escol hemos as primeiras por mera comodidade e fácil disponibilidade.

Extensa revisão de literatura dos últimos 31 anos, evidenciou que o uso de antibióticoterapia profilática na cirurgia eletiva da hérnia inguinal (sem prótese), não traz nenhum beneficio aos pacientes ${ }^{51}$.

Nossos pacientes recebiam alta hospitalar no primeiro dia de pós-operatório, continuando em tratamento ambulatorial. Não notamos diferenças significantes na intensi dade da dor pós-operatória nos três grupos de herniorrafia.

Dentre as complicações imediatas, assinalamos os cinco casos que evol uíram para abscesso, necessitando de drenagem. Existe uma crença generalizada, que a infecção aumenta o risco de recidiva. Dos cinco casos de infecção grave, três evoluíram para recidiva (dois Bassini e um McVay), o que pode parecer à primeira vista um índice elevado. U ma análise mais cuidadosa, entretanto, revela que destes, só um (McVay) evoluiu para recidiva nos primeiros três meses de pós-operatório. As outras duas hérnias recidivaram após 1 a 3 anos, o que dificilmente pode ser imputado à necrose supurativa e deiscência precoce das suturas . Na realidade, na maioria das recidivas, outros fatores etiológicos são responsáveis pelo insucesso operatório ${ }^{52,53}$.

A análise dos resultados tardios das operações de hérnia, esbarra sempre numa dificuldade universal que é o tempo de seguimento. Só $25 \%$ das recidivas se mani festam durante o primei ro ano de pós-operatório e $50 \%$ só aparecem após mais de cinco anos ${ }^{54}$. Rutledge informa que o tempo médio de aparecimento das reci divas é de 11,8 anos após a cirurgia original ${ }^{27}$. Recidivas avaliadas com seguimento inferior a 10 anos são incuráveis segundo alguns ${ }^{55}$. Ora, é praticamente impossível examinar-se periodicamente, durante tanto tempo, pacientes operados, principalmente em grandes coletivos. As pessoas mudam de resi dência com freqüência e isto faz com que se lance mão de tel efonemas e cartas, cujas informações não são confiáveis ${ }^{54,56,57}$.

Outro problema são os tempos de seguimento variáveis num coletivo a ser analisado. Em nossas avaliações (tabela 2) observamos que 32 hérnias só foram seguidas por seis meses. O que aconteceu com as mesmas após este período? Se calcularmos o grau de insucesso na nossa casuística, só levando em conta as 13 recidivas (num total de 136 hérnias), obteríamos uma taxa de $9,55 \%$ de recorrência, o que entretanto não corresponde à realidade. Infelizmente é desta forma que se calculam tais índices na maioria dos 
trabalhos publicados. Estes fatos explicam a grande divergência de informações na literatura.

Estudo populacional conduzido pela Rand Corporation, em 1983, informa que o índice geral de reci diva das hérnias inguinais operadas nos EUA é de no mínimo $10 \%{ }^{54}$. No Reino Unido, em centros não especializados, varia entre $5 \%$ e $20 \%{ }^{58}$. Na Alemanha no ano de 1992 era de $15 \%$ a $20 \%{ }^{57}$. Os índices reais de recidiva, entretanto, são provavel mente mai ores do que os relatados. Outra fonte de erro são os estudos retrospectivos que não têm a mesma acurácia das análises prospectivas.

É necessário, portanto, que se procure auxílio na estatística. Dos diferentes métodos estatísticos à disposição, o de Kaplan-Mayer, com avaliações actuariais, parece ser o melhor ${ }^{41,59-63}$. Permite que se preveja com bastante precisão o que irá acontecer futuramente mesmo com um seguimento incompleto de todos os casos.

Os índices de recorrência publicados pela Clínica Shouldice, de 0,7\% a 1,6\%, não são reproduzíveis em outros centros de Cirurgia Geral, nos quais análises clínicas comparativas entre diferentes técnicas têm apontado taxas da ordem de 3,6\% ${ }^{56}, 3,8 \%{ }^{57}, 4,0 \%{ }^{64} \mathrm{e}$ $10,8 \%{ }^{65}$. N osso índice de $23,7 \%$ não encontra similar na literatura. De qualquer forma, não houve diferença estatisticamente significativa entre este val or e o de 8,5\% encontrado para a técnica de McVay.

Para a técnica de Bassini, trabalhos retrospectivos tanto quanto prospectivos, apontam para indices de recidiva variando entre extremos de $1,8 \%{ }^{64}$ e $35,1 \%{ }^{57}$, passando por valores intermediários como $8,6 \%^{41}, 10,9 \%^{66}, 14,3 \%^{65}$ e $21,3 \%^{39}$. Nosso índice de $35,7 \%$, após quatro anos, situa-se entre os mais altos referidos, mas não surpreende se compararmos com o índice de 35,1\% após dois anos em um trabalho retrospectivo de Mückter ${ }^{57}$.

Rutledge 27,33 tem talvez a mai or experiência com a técnica de McVay, tendo operado 1.142 hérnias entre 1959 e 1991, em pacientes de ambos os sexos. Relata uma taxa de recidiva gl obal de $2 \%$ que caiu para $0,13 \%$ quando deixou de transplantar o funículo espermático para a tela sub-cutânea (acompanhamento médio de 7,4 anos).

Outras observações clínicas relatam índices entre $4,9 \%{ }^{67}, 11,2 \%{ }^{41}$ e $19,3 \%{ }^{63}$. Nossa taxa de $8,5 \%$ projetada para quatro anos de seguimento está dentro dos limites informados mundial mente.

Outro estudo, usando como nós o teste de KaplanMayer e curvas actuariais para 10 anos, porém retrospectivo, informa índice de recidiva de $19,3 \%$ para hérnias indiretas operadas pela técnica de McVay ${ }^{63}$. Paul et al. ${ }^{39}$ comparando Bassini vs Shouldice relata índices de 9,6\% para Bassini contra 1,7\% para Shouldice em 3,3 anos de seguimento e valores de $21,3 \%$
(Bassini) e 3,8\% (Shouldice) projetados para 20 anos.

B eets et al. ${ }^{68}$ em estudo recente, randomizado, utilizando cálculos estatísticos de sobrevida para 12 e 15 anos, informa índices de reci diva de 32\% a $33 \%$ para Bassini e 34\% para o estreitamento isol ado do anel inguinal profundo. Tais resultados foram significati vamente piores do que os observados com a técnica de Shouldice (15\%).

Analisando-se nossos resultados, podemos observar que o método de Bassini trouxe os piores resultados tardios. Das 13 recidivas observadas, oito se situavam no grupo Bassini. Observando-se a tabela 3, verifica-se que esta técnica foi particularmente mórbida nas hérnias diretas $(29 \%$ de recorrência).Das oito hérnias operadas a Bassini que recidivaram e das duas operadas a McVay, só uma de cada grupo submeteu-se à reoperação. Em ambas pudemos verificar a destrui ção quase completa da parede posteri or do conduto inguinal. Das três hérnias recidivadas no grupo Shouldice, uma foi reoperada e o que observamos foi somente um al argamento do anel inguinal profundo dando saída à gordura pré-peritoneal. Todas foram tratadas através da colocação de próteses de márlex, pela técnica de Stoppa ${ }^{69}$ no caso Bassini e pela técnica da "tela-rolha"70-72 nas outras duas.

Das 13 recidivas, oito ocorreram dentro dos primeiros 6 a 12 meses após a herniorrafia e destas, seis pertenciam ao grupo Bassini. Outras cinco recidivas (duas Bassini, duas Shouldice e uma McVay) só aconteceram após três anos. As recidivas precoces são conseqüência indiscutível de insuficiência técnica e é onde encontramos a maioria das herniorrafias a Bassini recidivadas. Já as recidivas tardias devem-se, na sua maior parte, à alterações degenerativas progressivas do tecido conectivo da região ${ }^{3}$, próprias das pessoas na segunda metade da vida. Nossos pacientes deste grupo tinham todos idades acima de 45 anos.

Aplicando-se o teste de Kaplan-M ayer com curvas actuariais de sobrevida, observamos resultados tardios estatísticamente significantes quando comparamos o método de Bassini isoladamente com as técnicas de Shouldice (Figura1) e McVay (F igura 2). Numa projeção de quatro anos de seguimento, 91,5\% dos pacientes operados a McVay e $76,3 \%$ dos operados a Shouldice permanecem sem recidiva, comparados com os $64,3 \%$ dos operados a Bassini. E m outras palavras, os índices de recidivas projetados actuarialmente para quatro anos, são de $35,7 \%$ para as hérnias operadas a Bassini, 23,7\% para Shouldice e $8,5 \%$ para McVay (Figura 4). As diferenças observadas entre Shouldice e McVay não são significativas ( $p=0,374$ - Figura 3 ).

Acredita-se que haja uma maior propensão de 
reci diva nas hérnias bilaterais, devi do ao aumento de tensão gerado nas suturas de aproximação músculo-ligamentares utilizadas nas técnicas aqui anal isadas ${ }^{73}$. N ossas obser vações entr etanto não apontam para diferenças significantes nos índices de recidiva das hérnias unilaterais quando comparadas com as bilaterais. Calculando-se através de curvas de sobrevida de Kaplan-Mayer as porcentagens de pacientes sem recidiva nas três técnicas, considerando-se separadamente as hérnias unilaterais e as bilaterais (Tabela 4), não pudemos observar diferenças estatisticamente significantes entre elas. Assim, nas técnicas de Bassini e McVay, as porcentagens de pacientes operados sem recidiva após quatro anos, são praticamente as mesmas nos pacientes com hérnias unilaterais e bilaterais. $\mathrm{Na}$ técnica de Shouldice estas comparações ficam prejudicadas pois temos apenas 25 meses de seguimento máximo nas hérnias bilaterais, quando não tínhamos nenhuma recidiva. Neste período observamos $90 \%$ de pacientes sem recidiva nas hérnias unilaterais.

Com a variedade de métodos operatórios para correção de hérnias inguinais à disposição dos cirurgiões nos dias de hoje, fica difícil indicar diretrizes normativas. O princípio das técnicas atensionais "tension-free", pelo qual se evitam suturas aproximativas de tecidos, colocando-se uma tela como anteparo e refor ço, seja por via anterior ou por via posterior ${ }^{74}$, veio revolucionar o tratamento das hérnias inguinais nos últimos anos. O menor desconforto pós-operatório e a mais rápida recuperação destes pacientes éum fato de fácil evi dência. Os resultados tardios carecem ainda de uma análise segura pelo pouco tempo de seguimento. Os custos e a morbidade destes processos podem ser maiores do que nas técnicas "convencionais". Acrecente-se a isto a maior curva de aprendizado, pel o menos para as técnicas Iaparoscópicas. Acreditamos que o uso de próteses não é necessário e mesmo desaconseIhável em pacientes jovens e em mulheres nos quais predominam hérnias indiretas pequenas. As técnicas abertas, ditas "convencionais", não estão proscritas, mas ao escol her uma delas, as nossas observações sugerem que a de Bassini deva ser evitada pelo elevado índice de insucesso a longo prazo. Portanto, respondendo à indagação inicial, título deste trabalho, respondemos que a técnica de Bassini não é mais atual, não negando-lhe o mérito históri co de ter si do o método operatóri o dominante por mais de um século.

\section{SUMMARY}

Treatment of inguinal hernias. A prospective,

\section{randomized trial comparing theree operative techniques: Bassini, Shouldice and McVay}

OBJ ECTIVE. to compare late results (recurrence) of three different techniques for treatment of inguinal hernias in theadult: Bassini, Shouldiceand McVay.

Patients AND Methods. theoperativelateresults of three surgical techniques: Bassini, Shouldice and McVay in 119 adult patients with inguinal hernias (some with bilateral pathology, totalizing 136 hernias) were analyzed. The majority of patients were males (93.3\%). The analysis was prospective, randomized, with uniform distribution of all three types of inguinal hernia (direct, indirect and combined) among the three groups of operative techniques. The number of recurrences was submitted to an actuarial analysis for a period of 4 years. The results underwent statistical analysis by the Kaplan-Mayer test with actuarial survival curves.

RESULTS. Eight hernia operations by the Bassini technique recurred in this time span, 3 in the Shouldice group and 2 in McVay. Among the Bassini recurrences, the worst results were observed with direct hernias (29\% recurrence) when compared with indirect ones (16\% recurrence). Overall recurrence rates plotted in an actuarial survival curve for 4 years, revealed statistically significant differences between Bassini and Shouldice: $35.7 \%$ versus $23.7 \%$; the same happened when comparing Bassini to McVay: $35.7 \%$ versus $8.5 \%$. The differences between Shouldice and McVay were not significant.

CONCLUSION. a recurrence rate of $35,7 \%$ for inguinal herniorraphy with the Bassini technique in a General Surgery University Clinic was surprising and obliged us to interrupt the trial. Our observations point to a prohibitive high failure rate when dealing with the Bassini technique, which was, over a century, the most popular treatment of inguinal hernia all over the world. Shouldice and McVay techniques, even though more complex, should be preferred whenever one makes the choice for "conventional" hernia treatment. [Rev Ass Med Brasil 1999; 45(2): 105-14.]

KEY WORDS. Hernia. Inguinal, hernia.

\section{REFERÊNCIAS BIBLIOGRÁFICAS}

1. Fruchaud $H$. Anatomie chirurgicale des hernies de l'aine. Paris,G.Doin \& Cie, 1956.

2. Peacock E E J r. Biology of hernia. In Nyus L M, Condon RE eds. Hernia, 2 nd ed. Philadel phia, Toronto, J .B. Lippincot Company, 1978; 79-97.

3. Peacock EE J r., Madden JW. Studies on the biology and treatment of recurent inguinal hernia: morfological changes. Ann Surg 1974; 179: 567-571. 
4. Ajabnoor MA, Mokhtar AM, Rafee AA, Taha AM. Defective collagen metabolism in Saudi patients with hernia. Ann Clin Biochem 1992; 29: 430-435.

5. Rodrigues J R. AJ , de Tolosa ECM, Carvalho CAF. Electron microscopic study on the elastic and elastic related fibers in the human fascia transversalis at different ages. Gegenbaurs Morphol J ahrbl 1990; 136: 645-650.

6. Rodrigues J r. AJ , Rodrigues CJ , Cunha ACP, Mittelstaedt WE M. Quantitative changes in the collagen and elastic fibers of the fascia transversalis from patients with direct and indirect inguinal hernia. In Abstract, Annual Meeting of the American Association of Clinical Anatomists. Hawai.USA, 1997;p.66.

7. Read,RC.The matabolic role in the attenuation of transversalis fascia found in patients with groin herniation. In Abstract. American Hernia Society. Anual Meeting. Miami.Florida.1998.Feb.6-8.

8. Griffith CA. The Marcy repair of indirect inguinal hernia: 1870 tothepresent. I n Nyhus LM , Condon RE eds. Hernia, 4th ed. Philadelphia, J B Lippincott Company, 1995; 111-112.

9. Bassini E. Über dieBehandlung des Leistenbruches. Langenbechs Arch Chir 1890; 40: 429-476.

10. Lichtenstein IL. Herniorrhaphy. A personal experience with 6,321 cases. AmJ Surg 1987; 153: 553-59.

11. Vogt DM, Curet MJ, Pitcher DE, Martin DT, Zucker KA. Preliminary results of a prospective randomized trial of laparoscopic onlay versus conventional inguinal herniorrhaphy. Am J Surg 1995; 169: 84-90.

12. Usher FC. Further observation on the use of marlex mesh. A new technique for the repair of inguinal hernias. Am Surg 1959; 25 (10) : 792-795.

13. U sher FC. Hernia repair with marlex mesh. Arch Surg 1962; 84: 325-328.

14. Usher FC. Hernia repair with knitted polypropylene. Surg Gynecol Obstet 1963; 117 (2): 239-240.

15. Falci $F$. Marlex mesh no tratamento da hérnia inguinal do adulto (Análise de 100 casos operados). O Hospital 1969; 75 (1): 161-173.

16. Falci $F$, Rezende $A O$. Metodização técnica da reparação da hérnia inguinal direta. Rev Col Bras Cir 1976; 3 (2): 85-90.

17. Nyhus LM. Individualization of hernia repair: a new era. Surg 1993; 114 (1): 1-2.

18. Ponka J L. The hernia problem in the female. In Ponka J $L$ ed. Hernias of theabdominal wall, Philadel phia, London, Toronto, WB Saunders Company, 1980; 82-90.

19. Andrews NJ . Presentation and outcomeof strangulated external hernia in a district general hospital. BrJ Surg 1981; 68: 329-332.

20. Lex A, Valtorta A. Hernias Inguinais. In Lex A, Valtorta A eds. Hernia, São Paulo, Panamed E ditorial, 1984; 33-93.

21. Bassini E. Sulla cura radicale dell'ernia inguinale. Arch $\mathrm{Soc}$ I tal Chir 1887; 4: 380.

22. Bassini E. Sopra 100 casi di cura radicale dell'ernia inguinale operata col metodo dell autore. Arch ed Atti d Soc I tal di Chir. 1888; 5: 315-319.

23. Bassini E. Nuovo metodo per la cura radicale dell'ernia inguinale. Padua, I taly. Prosperini, 1989.

24. Bassini E. Neue Operationsmethode zur Radikalbehandlung der Schenkel hernie. Langenbecks Arch Chir 1894; 47: 1-15.

25. Wantz GE. The operation of Bassini as described by Attilo Catterina. Surg Gynecol Obstet 1989; 168: 67-80 .

26. Anson BJ , Morgan EH, Mc Vay CB. Surgical antomy of the inguinal region based upon a study of 500 body halves. Surg Gynecol Obstet 1960; 111: 707-725.

27. Rutledge RH. Cooper's ligament repair: a 25 year experience with a singletechniquefor all groin hernias in adults. Surgery 1988; 103: 1-10.
28. Lichtenstein IL, Amid PK, Shulman AG. The ileopubic tract. Thekey toinguinal herniorrhaphy. Int Surg 1990; 75: 244-246.

29. Lotheissen G. Zur Radikalbehandlung der Shenkelhernien. Zentralbl Chir 1898; 25: 548-556.

30. Mc Vay CB, Anson BJ. A fundamental error in current methods of inguinal herniorrhaphy. Sur Gynecol Obstet 1942: 74: 746-750. tt, 1989; 119-136.

33. Rutledge RH. The Cooper ligament repair. Surg Gynecol Obstet 1993; 73 (3): 471-485.

34. Barbier J , Caretier J P. Cooper ligament repair: an update. World J Surg 1989;13: 499-505.

35. Nyhus LM. Editors Comment. In Nyhus LM, Condon RE eds. Hernia, 4th ed. Philadel phia, J .B. Lippincott, 1995;134-135.

36. Wantz GE. The Canadian Repair: personal observations. World J Surg 1989; 13: 516-521.

37. Moran RM, Blick M, Collura M. Doublelayer of transversalis fascia for repair of inguinal hernia. Results in 104 cases. Surgery 1968; 63: 423-430.

38. Sheaburn EW, Myers RN. Shouldice repair for inguinal hernia. Surgery 1969; 66: 450-460.

39. Paul A, Troidl H, William J I, Rixen D, Langen R. Randomized trial of modified Bassini versus Shouldice inguinal hernia repair. Brit J Surg 1994; 81: 1531-1534.

40. Simon MP, Kleijnen J , van GeldeneD, Hoitsma HFW, Obertop $\mathrm{H}$. Role of the Shouldice technique in inguinal hernia repair: a systematic review of controlled trials and a meta-analysis. Brit J Surg 1996; 83: 734-738.

41. Hay J M, Boudet MJ , Fingerhut $A$ et al. Shouldice inguinal repair in the male adult; the Gold Standard? Ann Surg 1995; 222 (6): 719-727.

42. Glasow $F$. The surgical repair of inguinal and femoral hernias. Can Med AssocJ 1973; 108: 308-313.

43. Bendavid R. The Shouldice method of inguinal herniorrhaphy. In Nyhus LM, Condon J B eds. Hernia 4th ed. Philadelphia, J .B. Lippincot, 1995; 217-236.

44. Bendavid R. N ew techniques in hernia repair. World J Surg 1989;13: 522-531.

45. Welsh DRJ ,Alexander MAJ . TheShouldiceRepair. SurgClin North Am 1993; 73 (3): 451-469.

46. Glassow F. Inguinal hernia repair. A comparison of the Shouldice and Cooper ligament repair of the posterior inguinal wall. AmJ Surg 1976; 131: 306-311.

47. Wantz GE. The Canadian Repair of inguinal hernia. In Nyhus LM, Condon RE eds. Hernia 3rd ed. Philadelphia, J B Lippincot, 1989; 236-252.

48. Berliner SD. A n approach to groin hernia. Surg Clin North Am 1984; 64: 197-213.

49. Mansberger J A, Rogers DA, J ennings WD, Leroy J . A comparison of a new two-layer anatomic repair to the traditional Shouldice herniorrhaphy. Am Surg 1992; 58: 211-212.

50. Kux M, Fuchsjäger N, Feichter N. Lichtenstein patch versus Shouldice Technique bei primären Leistenhernien mit hoher Rezidivgefährdung. Chirurg 1994; 65: 59-62.

51. Cheek C. A systematic review on the use of prophylactic antibiotics in groin hernia repair. In Abstract, American Hernia Soci ety. Anual Meeting. Miami. Florida.1998.F eb. 6-8.

52. Glassow F. I s postoperative wound infection following simple inguinal herniorrhaphy a predisposing cause of recurrent hernia? Canad Med Ass J 1964; 91: 870-871.

53. Lichtenstein IL, Shore J M. Exploding the myths of hernia repair. AmJ Surg 1976; 132: 307-315.

54. Lichtenstein IL, Shulman AG, Amid PK. The cause, prevention and treatment of recurrent groin hernia. Surg Clin N orth Am 1993; 73 (3): 529-544.

55. Glassow F. Recurrent inguinal and femoral hernia. Brit Med J 1970; 1: 215-219. 
56. Kux M, Fuchsjäger $N$, Schemper $M$. Shouldice is superior to Bassini inguinal herniorrhaphy. AmJ Surg 1994; 168: 15-18.

57. Mückter $H$, Reuters $G$, Vogel W. Leisenhernienreparation nach Bassini und nach Shouldice. Chirurg 1995; 65: 121-126.

58. Morgan M, Reynolds A, Swan AV, Beech R, Devlin HB. Are current techniques of inguial hernia repair optimal ? A survey in the United Kingdom. Ann R Coll Surg Engl 1991; 73: 341-345.

59. Kleinmuntz B. Clinical and actuarial judgement. Science 1990; 247: 146-147.

60. Dawes R, Faust D, Meehl PE. Clinical versus actuarial judgement. Science 1989; 243: 1668-1673.

61. Worthington GS J r. How to evaluate a surgical article: how much do "statistic"help? Current Surgery 1988; 45 (4) : 274-276.

62. Fallon WM. A historical perspective of statistics. Mayo Clinic Proc. 1988; 63: 952-954.

63. Asmussen T, J ensen FU. A follow-up study on recurrence after inguinal hernia repair. Surg Gynecol Obstet 1983; 156: 198-200.

64. Kingsnorth A N , Gray MR, N oth DM. Prospective randomized trial comparing the Shouldice technique and plication darn for inguinal hernia. Br J Surg 1992; 79: 1068-1070.

65. Tran VK, Pütz T, Rohde H. A randomized controlled trial for inguinal hernia repair to compare the Shouldice and BassiniKirchner Operation. Int Surg 1992; 77: 235-237.

66. Hoffmann R, Attinger TF B, Platz A, Largiader F. Bassini oder Shouldice? Helv Chir Acta 1991; 58: 207-212.
67. Halverson K, Mc Vay CB. Inguinal and femoral hernioplasty. A 22 year study of the authors methods. Arch Surg 1970; 101:127-131.

68. Beets GL, Oosterhuis KJ , GoPMNYH, et al. Long term followup (12-15 years) of a randomized controlled trial comparing Bassini-Stetten, Shouldice and high ligation with narrowing of the internal ring for primary inguinal hernia repair. In Abstract, American Hernia Society. Anual Meeting. Miami. Florida. 1998. Feb 6-8.

69. Stoppa RE. The preperitoneal approach and prosthetic repair of groin hernias. I n Nyhus LM , Condon RE eds. Hernia 4th ed. Philadel phia, J .B. Lippincot Company, 1995;188-210.

70. Rutkow I, Robbins AW. Mesh plug hernia repair: a follow-up report. Surgery 1995; 117: 597-598.

71. Campanelly GP, Cavagnoli R, Gabrielli F, Pietri P. Trabuccos procedure and local anesthesia in surgical treatment of inguinal and femoral hernia. Int Surg 1995; 80: 29-34.

72. Gilbert AI. Sutureless repair of inguinal hernia. Am J Surg 1992; 163: 331-335.

73. Ponka J L. Recurrent direct inguinal hernias. In Ponka J L ed. Hernias of theabdominal wall. Philadel phia, London, Toronto. WB Saunders Company, 1980; 216-237.

74. Amid PK. Hernioplastia anterior sem tensão de Lichtenstein e sua comparação com as técnicas laparoscópicas de correção das hérnias inguinais. Bol Inf CBC 1998; 99: 50-57. 